Proceedings of the Edinburgh Mathematical Society (2007) 50, 1-21 (C)

DOI:10.1017/S0013091505000672 Printed in the United Kingdom

\title{
AUTOMORPHISMS OF ENDOMORPHISM MONOIDS OF RELATIVELY FREE BANDS
}

\author{
JOÃO ARAÚJOํ AND JANUSZ KONIECZNY² \\ ${ }^{1}$ Universidade Aberta, R. Escola Politécnica, 147, 1269-001 Lisbon, Portugal and \\ Centro de Álgebra, Universidade de Lisboa, 1649-003 Lisbon, \\ Portugal (mjoao@lmc.fc.ul.pt) \\ ${ }^{2}$ Department of Mathematics, University of Mary Washington, \\ Fredericksburg, VA 22401, USA (jkoniecz@umw.edu)
}

(Received 6 May 2005)

\begin{abstract}
For a set $X$ and a variety $\mathcal{V}$ of bands, let $B_{\mathcal{V}}(X)$ be the relatively free band in $\mathcal{V}$ on $X$. For an arbitrary band variety $\mathcal{V}$ and an arbitrary set $X$, we determine the group of automorphisms of $\operatorname{End}\left(B_{\mathcal{V}}(X)\right)$, the monoid of endomorphisms of $B_{\mathcal{V}}(X)$.
\end{abstract}

Keywords: free band; variety; endomorphism; automorphism

2000 Mathematics subject classification: Primary 20M07; 20M20; $20 \mathrm{M} 15$

\section{Introduction}

In general, given a variety $\mathcal{A}$ of algebras, it is of independent interest to determine the group of automorphisms of $\operatorname{End}(A)$, where $A$ is a free algebra in $\mathcal{A}$ and $\operatorname{End}(A)$ is the monoid of endomorphisms of $A$. Many years ago, Schreier [22] and Mal'cev [15] solved this problem for unstructured sets (that is, algebras without operations) and Gluskin [11] solved it for vector spaces. In recent years, this kind of question has gained in importance and attracted wider attention for its links to universal algebraic geometry. (For a detailed explanation of these links, a list of references and related problems, see the excellent paper by Mashevitzky et al. [17].) Given the large number of published papers, preprints, lectures and, especially, open problems that have recently appeared on this subjectprompted by the links to universal algebraic geometry - we can anticipate that, for years to come, many new papers will be written describing the group of automorphisms of $\operatorname{End}(A)$ for various varieties $\mathcal{A}$.

Along this line of research, our aim is to describe the automorphisms of $\operatorname{End}(A)$ for any free object $A$ of any variety $\mathcal{V}$ of bands (idempotent semigroups). Analogous problems dealing with varieties of semigroups were solved by Mashevitzky and Schein [16] for the variety of all monoids and by Formanek [8] for the variety of all groups. However, we solve the problem not only for the variety of all bands but also for all subvarieties of this 
variety. In fact, with the very particular exceptions of sets and vector spaces, there is no other variety of algebras for which this problem has been solved for all free objects of all subvarieties.

Varieties of bands and their free objects have been studied by numerous authors. The lattice of all varieties of bands was described by Birjukov [4], Fennemore $[\mathbf{6}, \mathbf{7}]$ and Gerhard [9] (see Figure 1 on p. 15). A simplified system of identities for band varieties (one identity for each variety if one excludes $x^{2}=x$ ) and a solution of the world problem in relatively free bands were given by Gerhard and Petrich [10].

For a non-empty set $X$ and a variety $\mathcal{V}$ of bands, denote by $B_{\mathcal{V}}(X)$ the relatively free band in $\mathcal{V}$ on $X$ and by $\operatorname{End}\left(B_{\mathcal{V}}(X)\right)$ the monoid of endomorphisms of $B_{\mathcal{V}}(X)$. To determine the group $\operatorname{Aut}\left(\operatorname{End}\left(B_{\mathcal{V}}(X)\right)\right)$, we use Theorem 2.1, which states that, for every band $B$, the automorphisms of $\operatorname{End}(B)$ are induced by permutations on $B$. This theorem and other preliminary material is presented in $\S 2$. In $\S 3$, we introduce the concept of a $d$-invariant relatively free band and determine $\operatorname{Aut}\left(\operatorname{End}\left(B_{\mathcal{V}}(X)\right)\right)$ for every band variety $\mathcal{V}$ that includes the variety $\mathcal{S L}$ of semilattices. This group is either $S(X) \times C_{2}$ or $S(X)$, depending on whether or not $B_{\mathcal{V}}(X)$ is $d$-invariant. Section 4 deals with the varieties of rectangular bands. It turns out that the variety $\mathcal{V}$ of all rectangular bands is exceptional in the sense that $\operatorname{Aut}\left(\operatorname{End}\left(B_{\mathcal{V}}(X)\right)\right)$ is not encountered in any other band variety. In $\S 5$, we determine which relatively free bands are $d$-invariant and which are not. This gives us corollaries that determine $\operatorname{Aut}\left(\operatorname{End}\left(B_{\mathcal{V}}(X)\right)\right)$ given any band variety $\mathcal{V}$ and any set $X$. Finally, $\S 6$ provides a number of open problems.

\section{Preliminaries}

Let $S$ be a semigroup. Any bijection $\phi: S \rightarrow S$ such that $(a b) \phi=(a \phi)(b \phi)$ for all $a, b \in S$ is called an automorphism of $S$. (We write mappings on the right, that is, $a \phi$ rather than $\phi(a)$.) The group of automorphisms of $S$ will be denoted by $\operatorname{Aut}(S)$. A subset $C$ of $S$ is called characteristic in $S$ if $C \phi=C$ for every $\phi \in \operatorname{Aut}(S)$, where $C \phi=\{a \phi: a \in C\}$.

Let $X$ be an arbitrary non-empty set. The full transformation semigroup $T(X)$ consists of all mappings from $X$ to $X$ (full transformations of $X$ ) with composition as the semigroup operation. The semigroup $T(X)$ has the symmetric group $S(X)$ of all permutations of $X$ as its group of units. Let $S$ be a subsemigroup of $T(X)$. An automorphism $\phi$ of $S$ is called inner if there exists a $g \in S(X)$ such that $a \phi=g^{-1} a g$ for every $a \in S$. (In such a case, we write $\phi=\phi_{S}^{g}$.) The set $\operatorname{Inn}(S)$ of all inner automorphisms of $S$ is a subgroup of $\operatorname{Aut}(S)$. We denote by $G_{S}$ the normalizer of $S$ in $S(X)$, that is, $G_{S}$ denotes the subgroup of the symmetric group $S(X)$ consisting of all permutations $g$ of $X$ such that $g^{-1} S g=S$. Note that every element $g \in G_{S}$ induces an inner automorphism $\phi_{S}^{g}$ of $S$.

A band is a semigroup in which every element is an idempotent. Let $B$ be a band. Define a ternary relation $\rho$ on $B$ by

$$
\rho=\{(x, y, z): z=x y\} .
$$

Since $x=x x$ for every $x \in B$, the relation $\rho$ is reflexive. It is clear that, for every mapping $a: B \rightarrow B, a$ is an endomorphism of $B((x y) a=(x a)(y a)$ for all $x, y \in B)$ if and only if 
$a$ preserves $\rho((x, y, z) \in \rho \Longrightarrow(x a, y a, z a) \in \rho$ for all $x, y, z \in B)$. Thus, $\operatorname{End}(B)$, the semigroup of endomorphisms of $B$, consists of all transformations of $B$ that preserve the reflexive relation $\rho$. It is well known (see [24, Theorem 1] or [3, Lemma 2.1]) that if $S$ is a semigroup of transformations of $X$ that preserve a given reflexive relation on $X$, then

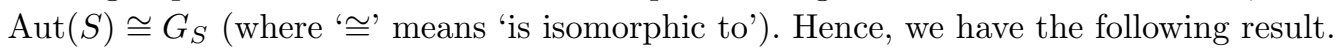

Theorem 2.1. Let $B$ be any band. Then

$$
\operatorname{Aut}(\operatorname{End}(B)) \cong G_{\operatorname{End}(B)} .
$$

We now introduce some standard material about band varieties in order to clearly establish notation and definitions. A variety of semigroups is a class of semigroups closed under subsemigroups, homomorphic images and direct products. Let $A$ be a countably infinite set. Denote by $A^{+}$the free semigroup on $A$. Let $u, v \in A^{+}$. We say that a semigroup $S$ satisfies the identity $u=v$ if $u \hat{\alpha}=v \hat{\alpha}$ for every $\alpha: A \rightarrow S$, where $\hat{\alpha}$ is the unique extension of $\alpha$ to a homomorphism from $A^{+}$to $S$. Let $R$ be a binary relation on $A^{+}$. We denote by $[R]$ the class of all semigroups $S$ such that $S$ satisfies the identity $u=v$ for every $(u, v) \in R$. If $R$ is finite, say $R=\left\{\left(u_{1}, v_{1}\right), \ldots,\left(u_{n}, v_{n}\right)\right\}$, we write $\left[u_{1}=v_{1}, \ldots, u_{n}=v_{n}\right]$ for $[R]$. Now, for every binary relation $R$ on $A^{+}$, the class $[R]$ is a variety of semigroups. Conversely, for every variety $\mathcal{V}$ of semigroups, there is a binary relation $R$ on $A^{+}$such that $\mathcal{V}=[R]$ (see $[\mathbf{1 3}$, p. 111]). For example, $[x y=y x]$ is the variety of commutative semigroups.

Let $\mathcal{V}$ be a non-trivial (containing a semigroup with at least two elements) variety of semigroups, and let $X$ be a non-empty set. We say that a semigroup $F \in \mathcal{V}$ is a $\mathcal{V}$-free semigroup on $X$ (or a relatively free semigroup in $\mathcal{V}$ on $X$ ) if it satisfies the following properties:

(1) $X$ generates $F$;

(2) for every $S \in \mathcal{V}$ and every mapping $\phi: X \rightarrow S$, there is an extension of $\phi$ to a homomorphism $\bar{\phi}: F \rightarrow S$.

Since $X$ generates $F$, an extension $\bar{\phi}$ is necessarily unique. For the variety $\mathcal{T}$ of trivial semigroups, we define a $\mathcal{T}$-free semigroup on $X$ to be any trivial semigroup. A $\mathcal{V}$-free semigroup on $X$ is isomorphic to $X^{+} / \rho_{\mathcal{V}}$, where $\rho_{\mathcal{V}}$ is the congruence on $X^{+}$defined by

$$
(u, v) \in \rho_{\mathcal{V}} \quad \Longleftrightarrow \quad \text { for every } S \in \mathcal{V} \text { and every } \alpha: X \rightarrow S, u \hat{\alpha}=v \hat{\alpha}
$$

(see $\left[\mathbf{1 3}\right.$, p. 110]). If $\mathcal{V}$ is a non-trivial variety, then $X$ is embedded in $X^{+} / \rho_{\mathcal{V}}$ via the injection $x \rightarrow x \rho_{\mathcal{V}}$ (where $x \in X$ and $x \rho_{\mathcal{V}}$ is the congruence class of $x$ modulo $\rho_{\mathcal{V}}$ ) [19, Lemma I.8.5]. Note that, for all varieties $\mathcal{V}_{1}$ and $\mathcal{V}_{2}$ of semigroups,

$$
\mathcal{V}_{1} \subseteq \mathcal{V}_{2} \Longleftrightarrow \rho_{\mathcal{V}_{2}} \subseteq \rho_{\mathcal{V}_{1}} .
$$

A variety of bands is a variety of semigroups in which every semigroup is a band. The band varieties form a lattice (under inclusion), with $\mathcal{B}=\left[x^{2}=x\right]$ as the greatest elements and $\mathcal{T}=[x=y]$ as the least element (see Figure 1). 
For a variety $\mathcal{V}$ of bands and a non-empty set $X$, we denote by $B_{\mathcal{V}}(X)$ the $\mathcal{V}$-free semigroup on $X$. We will refer to $B_{\mathcal{V}}(X)$ as the $\mathcal{V}$-free band on $X$, and assume that $B_{\mathcal{V}}(X)=X^{+} / \rho_{\mathcal{V}}$, where $\rho_{\mathcal{V}}$ is the congruence on $X^{+}$defined by (2.1). That is,

$$
B_{\mathcal{V}}(X)=\left\{w \rho_{\mathcal{V}}: w \in X^{+}\right\} .
$$

We will represent the congruence classes modulo $\rho_{\mathcal{V}}$ (the elements of $B_{\mathcal{V}}(X)$ ) by their representatives, that is, we will write $w \in B_{\mathcal{V}}(X)$ instead of $w \rho_{\mathcal{V}} \in B_{\mathcal{V}}(X)$. Moreover, for $u, v \in X^{+}$, we shall write $u=v$ both when $u \rho_{\mathcal{V}}=v \rho_{\mathcal{V}}$ (that is, when $u$ and $v$ are equal as elements of $\left.B_{\mathcal{V}}(X)\right)$ and when $u$ and $v$ are equal as words in $X^{+}$. It should always be clear from the context which equality is meant.

Let $\mathcal{V}$ be a non-trivial variety of bands. Since the mapping $x \rightarrow x \rho_{\mathcal{V}}$ from $X$ to $B_{\mathcal{V}}(X)$ is injective, we have the following:

$$
\text { for all } x, y \in X, \quad x=y \text { in } B_{\mathcal{V}}(X) \quad \Longleftrightarrow \quad x=y \text { in } X^{+} .
$$

Our objective is to determine the group of automorphisms of $\operatorname{End}\left(B_{\mathcal{V}}(X)\right)$, where $\mathcal{V}$ is an arbitrary variety of bands and $X$ is an arbitrary non-empty set. Almost all varieties of bands contain the variety of semilattices (see Figure 1). We begin our study with these varieties.

\section{Band varieties $\mathcal{V}$ with $\mathcal{S} \mathcal{L} \subseteq \mathcal{V}$}

A semilattice is a commutative band. The class $\mathcal{S} \mathcal{L}$ of semilattices is a variety. Throughout this section, we assume that $X$ is a non-empty set and that $\mathcal{V}$ is a variety of bands such that $\mathcal{S L} \subseteq \mathcal{V}$.

Our objective is to prove that $\operatorname{Aut}\left(\operatorname{End}\left(B_{\mathcal{V}}(X)\right)\right)$ is isomorphic to either $S(X)$ or $S(X) \times C_{2}$, where $C_{2}$ is the group with two elements. In view of Theorem 2.1, it suffices to prove the statement for $G_{\operatorname{End}(B)}$, where $B=B_{\mathcal{V}}(X)$. Recall that $G_{\operatorname{End}(B)}$ is the group of permutations $g \in S(B)$ such that $g^{-1} \operatorname{End}(B) g=\operatorname{End}(B)$.

For $w \in X^{+}$, the content $C(w)$ of $w$ is defined as the set of all elements of $X$ (letters) that occur in $w$. For example, if $w=a b a c a b c$, then $C(w)=\{a, b, c\}$. For the $\mathcal{S} \mathcal{L}$-free band $B_{\mathcal{S L}}(X)$, we have the following [13, Proposition 4.6.5]:

$$
\text { for all } u, v \in X^{+}, \quad u=v \text { in } B_{\mathcal{S L}}(X) \quad \Longleftrightarrow \quad C(u)=C(v) \text {. }
$$

Since $\mathcal{S} \mathcal{L} \subseteq \mathcal{V}$, it follows from (2.2) and (3.1) that

$$
\text { for all } u, v \in X^{+}, \quad u=v \text { in } B_{\mathcal{V}}(X) \quad \Longrightarrow \quad C(u)=C(v) \text {. }
$$

Let $x \in X$. It follows from (3.2) that $x \rho_{\mathcal{V}}=\left\{x^{k}: k \geqslant 1\right\}$, which we shall always represent by $x$.

Let $B=B_{\mathcal{V}}(X)$. We say that a subset $J$ of $B$ is independent if, for all $u, v \in J$, $u \neq v($ in $B \mathcal{V}(X)) \Longrightarrow C(u) \cap C(v)=\emptyset$. (Note that this definition makes sense because of (3.2).) If $J \subseteq B$ is not independent, we say that it is dependent.

Lemma 3.1. Let $B=B_{\mathcal{V}}(X)$ and $g \in G_{\operatorname{End}(B)}$. Then $X g=X$. 
Proof. Suppose $X$ is finite with, say, $n$ elements. Since $g$ is a bijection, $|X g|=n$. Observe that if $J \subseteq B$ is dependent and $a \in \operatorname{End}(B)$ restricted to $J$ is one-to-one, then $J a$ is dependent. (Indeed, if $u, v \in J$ with $u \neq v$ and $x \in C(u) \cap C(v)$, then $u a, v a \in J a$ with $u a \neq v a$ and $C(x a) \subseteq C(u a) \cap C(v a)$.)

Define $a \in \operatorname{End}(B)$ by $x a=x g^{-1}$ for every $x \in X$. (Such an $a$ exists and is unique since $B$ is the $\mathcal{V}$-free band on $X$.) For every $x \in X$,

$$
(x g)\left(g^{-1} a g\right)=x a g=x g^{-1} g=x .
$$

Thus, $(X g)\left(g^{-1} a g\right)=X$. It follows that $g^{-1} a g$ restricted to $X g$ is one-to-one (since $|X g|=|X|=n$ ). Hence, by the observation above, $X g$ must be independent (since $g^{-1} a g$ is an endomorphism and $X$ is independent). It follows that $X g=X$ (since $X$ is the only subset of $B$ that is independent and has $n$ elements).

Suppose $X$ is infinite. Let $x \in X$. We claim that $x g \in X$. Let $u=x g$ and suppose, by way of contradiction, that $u \notin X$. Then $u=p u^{\prime}$ for some $p \in X$ and $u^{\prime} \in B$.

Let $v=x g^{-1}$ and $w=p g^{-1}$. Then $w \neq x$ (since $w=x$ would imply $u=x g=w g=$ $\left(p g^{-1}\right) g=p \in X$, but we have that $\left.u \notin X\right)$. Since $X$ is infinite, there exists $z \in X$ such that $z \notin C(v)$. Define $a \in \operatorname{End}(B)$ by $x a=v$ and $y a=z$ for every $y \in X-\{x\}$. Then

$$
u\left(g^{-1} a g\right)=x(a g)=v g=x .
$$

Hence, since $g^{-1} a g \in \operatorname{End}(B)$, we have

$$
x=u\left(g^{-1} a g\right)=p\left(g^{-1} a g\right) u^{\prime}\left(g^{-1} a g\right) .
$$

Then $C\left(p\left(g^{-1} a g\right) u^{\prime}\left(g^{-1} a g\right)\right)=C(x)=\{x\}$, and so $p\left(g^{-1} a g\right)=u^{\prime}\left(g^{-1} a g\right)=x$. Thus, $x=p\left(g^{-1} a g\right)=\left(p g^{-1}\right) a g=(w a) g$, and so $w a=v$ (since $x=v g$ and $g$ is one-toone). Since $w \neq x$, there exists $y \in C(w)$ such that $y \neq x$. But then $z=y a$, and so $\{z\}=C(y a) \subseteq C(w a)=C(v)$, which is a contradiction (since $z \notin C(v)$ ).

The claim has been proved, that is, $X g \subseteq X$. By the foregoing argument applied to $g^{-1}$, we also have $X g^{-1} \subseteq X$. The result follows.

For a function $f: A \rightarrow B$ and $A_{1} \subseteq A$, we denote by $f \mid A_{1}$ the restriction of $f$ to $A_{1}$.

Lemma 3.2. Let $B=B_{\mathcal{V}}(X)$ and let $g \in G_{\operatorname{End}(B)}$ be such that $g \mid X=\mathrm{id}_{X}$. Then for every $w \in B, C(w g)=C(w)$.

Proof. Let $w \in B$. We first show that $C(w g) \subseteq C(w)$. Let $w=x_{1} \cdots x_{k}$ and $w g=$ $y_{1} \cdots y_{n}$, where $x_{i}, y_{j} \in X$. Define $a \in \operatorname{End}(B)$ by $x a=x$ for every $x \in C(w)$, and $z a=x_{1}$ for every $z \in X-C(w)$. Note that $C(u a) \subseteq C(w)$ for every $u \in B$. Now,

$$
\left(x_{1} \cdots x_{k}\right)\left(g g^{-1} a g\right)=\left(x_{1} \cdots x_{k}\right) a g=\left(\left(x_{1} a\right) \ldots\left(x_{k} a\right)\right) g=\left(x_{1} \cdots x_{k}\right) g=w g .
$$


On the other hand,

$$
\begin{aligned}
\left(x_{1} \cdots x_{k}\right)\left(g g^{-1} a g\right) & =\left(y_{1} \cdots y_{n}\right)\left(g^{-1} a g\right) \\
& =y_{1}\left(g^{-1} a g\right) \cdots y_{n}\left(g^{-1} a g\right) \quad\left(\text { since } g^{-1} a g \in \operatorname{End}(B)\right) \\
& =\left(y_{1} a g\right) \cdots\left(y_{n} a g\right) \quad(\text { since } g \mid X \text { is the identity) } \\
& =\left(y_{1} a\right) \cdots\left(y_{n} a\right) \quad \text { (since each } y_{j} a \in X \text { and } g \mid X \text { is the identity) } \\
& =\left(y_{1} \cdots y_{n}\right) a .
\end{aligned}
$$

Thus, $w g=\left(y_{1} \cdots y_{n}\right) a$, and so $C(w g)=C\left(\left(y_{1} \cdots y_{n}\right) a\right) \subseteq C(w)$.

By the foregoing argument applied to $g^{-1}$ and $w g$, we have $C(w)=C\left((w g) g^{-1}\right) \subseteq$ $C(w g)$. Hence, $C(w g)=C(w)$.

Let $S$ be a semigroup. A bijection $\phi: S \rightarrow S$ is called an anti-automorphism of $S$ if $(a b) \phi=(b \phi)(a \phi)$ for all $a, b \in S$.

For a word $w \in X^{+}$, the dual $\bar{w}$ of $w$ is the word obtained from $w$ by reversing the order of the letters. For example, if $w=c a b a c b a$, then $\bar{w}=a b c a b a c$. Note that, for all $u, v \in X^{+}$,

$$
\overline{\bar{u}}=u \quad \text { and } \quad \overline{u v}=\bar{v} \bar{u} .
$$

Let $B=B_{\mathcal{V}}(X)$. We say that $B$ is $d$-invariant if for all $u, v \in X^{+}$,

$$
u=v \text { in } B \quad \Longrightarrow \quad \bar{u}=\bar{v} \text { in } B .
$$

Let $B=B_{\mathcal{V}}(X)$ be $d$-invariant. The mapping $d: B \rightarrow B$ defined by $w d=\bar{w}$ will be called the duality mapping on $B$. Since $B$ is $d$-invariant, we have, for all $u, v \in B$, that

$$
u=v \quad \Longleftrightarrow \quad \bar{u}=\bar{v} \quad \Longleftrightarrow \quad u d=v d .
$$

Thus, $d$ is a well-defined bijection on $B$, that is $d \in S(B)$. It follows from the definition of $d$ and (3.3) that $d$ has the following properties:

(1) $d \mid X=\operatorname{id}_{X}$, that is, $x d=x$ for every $x \in X$;

(2) $d$ is an anti-automorphism of $B$, that is, $d$ is a bijection and $(u v) d=(v d)(u d)$ for all $u, v \in B$;

(3) $d=d^{-1}$, that is, $w(d d)=w$ for every $w \in B$.

Note that if $B=B_{\mathcal{V}}(X)$ and $d: B \rightarrow B$ given by $w d=\bar{w}$ is well defined, then $B$ is $d$-invariant.

We prove that if $B=B_{\mathcal{V}}(X)$ and $g \in G_{\operatorname{End}(B)}$, then $g$ is an automorphism of $B$ or an anti-automorphism of $B$ (Lemmas 3.3-3.7).

Lemma 3.3. Let $B=B_{\mathcal{V}}(X)$ be $d$-invariant and let $d$ be the duality mapping on $B$. Then $d \in G_{\operatorname{End}(B)}$. 
Proof. Since $d^{-1}=d$, it suffices to show that $d \operatorname{End}(B) d \subseteq \operatorname{End}(B)$. Let $a \in \operatorname{End}(B)$ and $u, v \in B$. Then

$$
(u v)(d a d)=(v d u d)(a d)=(v(d a) u(d a)) d=u(d a d) v(d a d) .
$$

Thus, $d a d \in \operatorname{End}(B)$, and the result follows.

In fact, using a similar argument, one can prove a more general statement: if $B=B_{\mathcal{V}}(X)$ and $g$ is an anti-automorphism of $B$, then $g \in G_{\operatorname{End}(B)}$.

Lemma 3.4. Let $B=B_{\mathcal{V}}(X)$, let $g \in G_{\operatorname{End}(B)}$ be such that $g \mid X=\operatorname{id}_{X}$, and let $x, y \in X$ with $x \neq y$. Then,

(1) if $(x y) g=x y$, then $g$ is the identity mapping on $B$,

(2) if ( $x y) g=y x$, then $g$ is the duality mapping on $B$.

Proof. Let $u$ and $v$ be arbitrary elements of $B$. Let $a \in \operatorname{End}(B)$ be such that $x a=u$ and $y a=v$. Then

$$
(x y)\left(g g^{-1} a g\right)=(x y)(a g)=(x a y a) g=(u v) g .
$$

To prove (1), suppose $(x y) g=x y$. Then, since $g^{-1} a g \in \operatorname{End}(B)$ and $g \mid X=\operatorname{id}_{X}$, we have

$$
(x y)\left(g g^{-1} a g\right)=(x y)\left(g^{-1} a g\right)=x\left(g^{-1} a g\right) y\left(g^{-1} a g\right)=x(a g) y(a g)=(u g)(v g) .
$$

Thus, $(u v) g=(u g)(v g)$, and so $g$ is an automorphism of $B$. But the only automorphism of $B$ that fixes every element of $X$ is $\operatorname{id}_{B}$. Hence, $g=\operatorname{id}_{B}$.

To prove (2), suppose that $(x y) g=y x$. Then, since $g^{-1} a g \in \operatorname{End}(B)$ and $g \mid X=\operatorname{id}_{X}$, we have

$$
(x y)\left(g g^{-1} a g\right)=(y x)\left(g^{-1} a g\right)=y\left(g^{-1} a g\right) x\left(g^{-1} a g\right)=y(a g) x(a g)=(v g)(u g) .
$$

Thus, $(u v) g=(v g)(u g)$, and so $g$ is an anti-automorphism of $B$. But the only antiautomorphism of $B$ that fixes every element of $X$ is $d$, the duality mapping on $B$. Hence, $g=d$.

Lemma 3.5. Let $B=B_{\mathcal{V}}(X)$, let $g \in G_{\operatorname{End}(B)}$ be such that $g \mid X=\mathrm{id}_{X}$, and let $x, y \in X$ with $x \neq y$. Then either $(x y) g=x y$ or $(x y) g=y x$.

Proof. By Lemma 3.2, $C((x y) g)=C(x y)=\{x, y\}$. Since $B$ is a band, the only elements of $B$ with content $\{x, y\}$ are $x y, y x, x y x$ and $y x y$.

Suppose $(x y) g=x y x$. Let $a \in \operatorname{End}(B)$ be such that $x a=x y$ and $y a=x$. Then

$$
(x y)\left(g g^{-1} a g\right)=(x y)(a g)=(x a y a) g=(x y x) g .
$$

On the other hand, since $(x y) g=x y x, g^{-1} a g \in \operatorname{End}(B)$, and $g \mid X=\operatorname{id}_{X}$, we have

$$
\begin{aligned}
(x y)\left(g g^{-1} a g\right) & =(x y x)\left(g^{-1} a g\right)=x\left(g^{-1} a g\right) y\left(g^{-1} a g\right) x\left(g^{-1} a g\right)=x(a g) y(a g) x(a g) \\
& =((x y) g)(x g)((x y) g)=(x y x) x(x y x)=x y x .
\end{aligned}
$$


Thus, $(x y x) g=x y x=(x y) g$, implying that $x y x=x y$ (since $g$ is one-to-one). Hence, $(x y) g=x y x=x y$.

Using a similar argument (with $a \in \operatorname{End}(B)$ such that $x a=y$ and $y a=x y$ ), we can prove that if $(x y) g=y x y$, then $y x y=x y$, and so $(x y) g=y x y=x y$.

Since the only remaining possibilities are $(x y) g=x y$ and $(x y) g=y x$, the result follows.

Lemma 3.6. Let $B=B_{\mathcal{V}}(X)$ and let $g \in G_{\operatorname{End}(B)}$ be such that $g \mid X=\operatorname{id}_{X}$. Then $g=\operatorname{id}_{B}$ or $g=d$.

Proof. If $|X|=1$, say $X=\{x\}$, then $B=\{x\}$, and so $g=\operatorname{id}_{B}$. If $|X| \geqslant 2$, then the result follows immediately from Lemmas 3.4 and 3.5.

Lemma 3.7. Let $B=B_{\mathcal{V}}(X)$ and $g \in G_{\operatorname{End}(B)}$. Then we have the following.

(1) If $B$ is $d$-invariant, then $g$ is an automorphism of $B$ or an anti-automorphism of $B$.

(2) If $B$ is not $d$-invariant, then $g$ is an automorphism of $B$.

(3) If $|X| \geqslant 2$ and $g$ is both an automorphism of $B$ and an anti-automorphism of $B$, then $\mathcal{V}=\mathcal{S} \mathcal{L}$.

Proof. To prove (1) and (2), define $\theta: G_{\operatorname{End}(B)} \rightarrow S(X)$ by $h \theta=h \mid X$. By Lemma 3.1, $\theta$ is well defined. Since $\left(h_{1} h_{2}\right) \mid X=\left(h_{1} \mid X\right)\left(h_{2} \mid X\right)$ for all $h_{1}, h_{2} \in G_{\operatorname{End}(B)}$, we find that $\theta$ is a group homomorphism.

Let $\alpha=g \theta$ (that is, $\alpha=g \mid X)$. Consider $h \in \operatorname{End}(B)$ defined by $x h=x \alpha$ for every $x \in$ $X$. Then $h$ is an automorphism of $B$. (Indeed, define $h^{\prime} \in \operatorname{End}(B)$ by $x h^{\prime}=x \alpha^{-1}$ for every $x \in X$. Then $x\left(h h^{\prime}\right)=x\left(h^{\prime} h\right)=x$ for every $x \in X$, which implies that $h h^{\prime}=h^{\prime} h=\mathrm{id}_{B}$.) Clearly, $h \theta=\alpha=g \theta$.

Suppose that $B$ is $d$-invariant. Then, by Lemma $3.6, \operatorname{Ker}(\theta)=\left\{\operatorname{id}_{B}, d\right\}$. Thus, since $h \theta=g \theta$, we have $g h^{-1} \in \operatorname{Ker}(\theta)=\left\{\operatorname{id}_{B}, d\right\}$, that is, $g=\operatorname{id}_{B} h$ or $g=d h$. In the former case, $g$ is an automorphism of $B$; and, in the latter case, it is an anti-automorphism of $B$. This proves (1).

Suppose that $B$ is not $d$-invariant. Then the duality mapping $d$ is not well defined on $B$, and so, by Lemma 3.6, $\operatorname{Ker}(\theta)=\left\{\operatorname{id}_{B}\right\}$. Thus, $\theta$ is one-to-one, and so $h \theta=g \theta$ implies $h=g$. Since $h$ is an automorphism of $B,(2)$ follows.

To prove (3), suppose $|X| \geqslant 2$ and $g$ is both an automorphism of $B$ and an antiautomorphism of $B$. Let $x, y \in X$. Since $g$ is onto, there exist $u, v \in B$ such that $x=u g$ and $y=v g$. Since $g$ is both an automorphism and an anti-automorphism, we have

$$
x y=(u g)(v g)=(u v) g=(v g)(u g)=y x .
$$

Thus, since $B$ is the $\mathcal{V}$-free band on $X$ and $|X| \geqslant 2$, it follows that, for every $S \in \mathcal{V}$ and for all $a, b \in S, a b=b a$. Hence, every band in $\mathcal{V}$ is a semilattice, which implies $\mathcal{V}=\mathcal{S} \mathcal{L}$. 
With the preceding lemmas, we can determine the group of automorphisms of the semigroup $\operatorname{End}\left(B_{\mathcal{V}}(X)\right)$ for any variety $\mathcal{V}$ of bands that contains the variety $\mathcal{S} \mathcal{L}$ of semilattices. We denote by $C_{2}$ the cyclic group of order 2 , say $C_{2}=\{1, t\}$.

Theorem 3.8. Let $X$ be a set with $|X| \geqslant 2$, let $\mathcal{V}$ be a variety of bands such that $\mathcal{S} \mathcal{L} \subseteq \mathcal{V}$, and let $B=B_{\mathcal{V}}(X)$. Then we have the following.

(1) If $\mathcal{V}=\mathcal{S L}$ or $B$ is not $d$-invariant, then $\operatorname{Aut}(\operatorname{End}(B))$ is isomorphic to $S(X)$.

(2) If $\mathcal{V} \neq \mathcal{S} \mathcal{L}$ and $B$ is $d$-invariant, then $\operatorname{Aut}(\operatorname{End}(B))$ is isomorphic to $S(X) \times C_{2}$.

Proof. Since Aut $(\operatorname{End}(B))$ is isomorphic to $G_{\operatorname{End}(B)}($ Theorem 2.1), $\operatorname{Aut}(\operatorname{End}(B))$ in statements (1) and (2) can be replaced with $G_{\operatorname{End}(B)}$.

To prove (1), suppose that $\mathcal{V}=\mathcal{S L}$ or $\mathcal{V}$ is not $d$-invariant. Define $\theta: G_{\operatorname{End}(B)} \rightarrow S(X)$ by $g \theta=g \mid X$. By the proof of Lemma 3.7, $\theta$ is a well-defined group homomorphism, $\operatorname{Ker}(\theta)=\left\{\operatorname{id}_{B}\right\}$, and for every $\alpha \in S(X)$ there exists an $h \in G_{\operatorname{End}(B)}$ such that $\alpha=h \theta$. (If $\mathcal{V}=\mathcal{S} \mathcal{L}$, then $\mathcal{V}$ is $d$-invariant, and so, according to the proof of Lemma 3.7, $\operatorname{Ker}(\theta)=$ $\left\{\operatorname{id}_{B}, d\right\}$. But if $\mathcal{V}=\mathcal{S} \mathcal{L}$, then $d=\mathrm{id}_{B}$, and so $\operatorname{Ker}(\theta)=\left\{\operatorname{id}_{B}\right\}$.) Hence, $\theta$ is a group isomorphism, which proves (1).

To prove (2), suppose that $\mathcal{V} \neq \mathcal{S L}$ and $\mathcal{V}$ is $d$-invariant. Define $\phi: G_{\operatorname{End}(B)} \rightarrow$ $S(X) \times C_{2}$ by

$$
g \phi= \begin{cases}(g \mid X, 1) & \text { if } g \text { is an automorphism of } B, \\ (g \mid X, t) & \text { if } g \text { is an anti-automorphism of } B .\end{cases}
$$

Then $\phi$ is well defined by Lemmas 3.1 and 3.7. It is routine to verify that $\phi$ is a group homomorphism. For example, suppose that $g_{1}, g_{2} \in G_{\operatorname{End}(B)}$ are anti-automorphisms of $B$. Then $g_{1} g_{2}$ is an automorphism of $B$, and so

$$
\left(g_{1} g_{2}\right) \phi=\left(\left(g_{1} g_{2}\right) \mid X, 1\right)=\left(\left(g_{1} \mid X\right)\left(g_{2} \mid X\right), 1\right)=\left(g_{1} \mid X, t\right)\left(g_{2} \mid X, t\right)=\left(g_{1} \phi\right)\left(g_{2} \phi\right) .
$$

Suppose $g \phi=\left(\operatorname{id}_{X}, 1\right)$. Then $g \mid X=\mathrm{id}_{X}$ and $g$ is an automorphism of $B$. Thus, $g=\operatorname{id}_{B}$. Hence, $\operatorname{Ker}(\phi)=\left\{\operatorname{id}_{B}\right\}$, and so $\phi$ is one-to-one.

Let $\alpha \in S(X)$. By the proof of Lemma 3.7, there is an automorphism $h$ of $B$ such that $h \mid X=\alpha$ and $h d \mid X=\alpha$. Hence, $h \phi=(\alpha, 1)$ and $(h d) \phi=(\alpha, t)$ (since $d$ is an anti-automorphism of $B$, and so $h d$ is an anti-automorphism of $B)$, which shows that $\phi$ is onto. Thus, $\phi$ is a group isomorphism, which proves (2).

\section{Varieties of rectangular bands}

A semigroup $S$ is called a rectangular band if $a b a=a$ for all $a, b \in S$. It is called a left zero semigroup if $a b=a$ for all $a, b \in S$, and it is called a right zero semigroup if $a b=b$ for all $a, b \in S$. It is clear that the left zero semigroups and right zero semigroups are rectangular bands.

The classes of all rectangular bands, left zero semigroups and right zero semigroups are varieties of bands, which we shall denote, respectively, by $\mathcal{R B}, \mathcal{L Z}$ and $\mathcal{R Z}$. These 
three varieties and the variety $\mathcal{T}$ of trivial bands are the only varieties of bands that do not contain the variety $\mathcal{S} \mathcal{L}$ of semilattices (see Figure 1).

Every left zero semigroup $B$ is relatively free in $\mathcal{L Z}$ on $X=B$. Since $a b=a$ for all $a, b \in B$, every transformation of $B$ is an endomorphism, that is, $\operatorname{End}(B)=T(B)$. It is well known $[\mathbf{1 5}, \mathbf{2 2}]$ that $\operatorname{Aut}(T(B)) \cong S(B)$. The same argument applies to right zero semigroups. It follows that if $\mathcal{V}=\mathcal{L Z}$ or $\mathcal{V}=\mathcal{R} \mathcal{Z}$, then $\operatorname{Aut}\left(\operatorname{End}\left(B_{\mathcal{V}}(X)\right)\right)$ is isomorphic to $S(X)$.

It remains to consider the variety $\mathcal{R B}$ of all rectangular bands. Every rectangular band $B$ is isomorphic to a semigroup $L \times R$, where $L=B z$ and $R=z B$ (for a fixed $z \in B$ ), with multiplication $\left(k_{1}, p_{1}\right)\left(k_{2}, p_{2}\right)=\left(k_{1}, p_{2}\right)$ [13, Theorem 1.1.3]. The monoid $\operatorname{End}(B)$ is isomorphic to $T(L) \times T(R)$. The following lemma shows that if $B$ is a $\mathcal{V}$-free band on $X$, then $B=X \times X$.

Lemma 4.1. Let $\mathcal{V}=\mathcal{R B}, B=B \mathcal{V}(X)$, and $z \in B$. Then

(1) $B z=X z$ and $z B=z X$,

(2) $|X z|=|z X|=|X|$.

Proof. To prove (1), let $w z \in B z$, where $w=x_{1} \cdots x_{n} \in B\left(n \geqslant 1, x_{i} \in X\right)$. Since $B$ is a rectangular band, $u v p=u p$ for all $u, v, p \in B$ [13, Theorem 1.1.3]. Thus, $w z=x_{1} \cdots x_{n} z=x_{1} z \in X z$. Hence, $B z=X z$. By a similar argument, we obtain $z B=z X$.

To prove (2), define $f: X \rightarrow X z$ by $x f=x z$. It is clear that $f$ is onto. Let $x, y \in X$ with $x \neq y$. Consider the rectangular band $L \times R$ with $L=\{1,2\}$ and $R=\{1\}$. Since $B$ is the $\mathcal{R B}$-free band on $X$, there exists a homomorphism $\phi: B \rightarrow L \times R$ such that $x \phi=(1,1)$ and $y \phi=(2,1)$. We have $z \phi=(a, 1)$, where $a=1$ or $a=2$, and so

$$
(x z) \phi=(x \phi)(z \phi)=(1,1)(a, 1)=(1,1) \quad \text { and } \quad(y z) \phi=(y \phi)(z \phi)=(2,1)(a, 1)=(2,1) .
$$

It follows that $x z \neq y z$, and so $f$ is one-to-one. Hence, $|X z|=|X|$. By a similar argument, we obtain $|z X|=|X|$.

We first consider $B=X \times X$ with $|X|=2$, say $X=\{1,2\}$. Here we use the fact that $\operatorname{Aut}(\operatorname{End}(B)) \cong G_{\operatorname{End}(B)}$. Let $a: B \rightarrow B$. We say that a left-splits $k_{0} \in X$ if there exist $p_{1}, p_{2} \in X$ such that $\left(k_{0}, p_{1}\right) a=\left(k_{1}, q_{1}\right)$ and $\left(k_{0}, p_{2}\right) a=\left(k_{2}, q_{2}\right)$ with $k_{1} \neq k_{2}$. We have a dual definition of what it means that a right-splits $p_{0} \in X$. It follows from $[\mathbf{1 3}$, Proposition 4.4.2] that $a \in \operatorname{End}(B)$ if and only if there exist $a_{1}, a_{2} \in T(X)$ such that $a=a_{1} \times a_{2}$. (For $f: A \rightarrow B$ and $g: C \rightarrow D$, we denote by $f \times g$ the mapping from $A \times C$ to $B \times D$ defined by $(x, y)(f \times g)=(x f, y g)$.) The existence of such $a_{1}$ and $a_{2}$ is clearly equivalent to $a$ not (left- or right-) splitting any element of $X$.

Let $g \in G_{\operatorname{End}(B)}$. (We continue to assume that $X=\{1,2\}$.) Suppose that $g$ left-splits 1, that is,

$$
(1,1) g=(k, p) \quad \text { and } \quad(1,2) g=(l, q)
$$


with $k \neq l$. We claim that $p=q$. Suppose $p \neq q$. Then either

(i) $(2,1) g=(k, q)$ and $(2,2) g=(l, p)$ or

(ii) $(2,1) g=(l, p)$ and $(2,2) g=(k, q)$.

Suppose that (i) is true. Consider $a=a_{1} \times a_{2} \in \operatorname{End}(B)$, where $1 a_{1}=2 a_{1}=1,1 a_{2}=1$, and $2 a_{2}=2$. Then $(k, p)\left(g^{-1} a g\right)=(k, p)$ and $(l, p)\left(g^{-1} a g\right)=(l, q)$. Thus, $g^{-1} a g$ rightsplits $p$, which is a contradiction since $g^{-1} a g \in \operatorname{End}(B)$. If (ii) is true, we obtain a contradiction in a similar way. Hence,

$$
(1,1) g=(k, p), \quad(1,2) g=(l, p), \quad(2,1) g=(m, q) \quad \text { and } \quad(2,2) g=(n, q),
$$

where $k \neq l, m \neq n$ and $p \neq q$. It follows that if $g$ left-splits 1 , then it left- and rightsplits 1 and 2 , and $(\{1\} \times X) g=X \times\{p\}$. We can use a similar argument when $g$ right-splits 1 , and when $g$ left- or right-splits 2 , obtaining the following lemma.

Lemma 4.2. Let $X=\{1,2\}, B=X \times X$, and $g \in G_{\operatorname{End}(B)}$. If $g$ splits any element of $X$, then, for every $i \in X$,

$$
(\{i\} \times X) g=X \times\left\{p_{i}\right\} \quad \text { and } \quad(X \times\{i\}) g=\left\{k_{i}\right\} \times X
$$

Lemma 4.3. Let $X=\{1,2\}, B=X \times X$ and $g: B \rightarrow B$. Then $g \in G_{\operatorname{End}(B)}$ if and only if there exist $g_{1}, g_{2} \in S(X)$ such that $g=g_{1} \times g_{2}$ or $g=\left(g_{1} \times g_{2}\right) \delta$, where $\delta \in S(B)$ is defined by $(k, p) \delta=(p, k)$.

Proof. It is clear that if $g=g_{1} \times g_{2}$ or $g=\left(g_{1} \times g_{2}\right) \delta$, then $g \in G_{\operatorname{End}(B)}$. To prove the converse, suppose $g \in G_{\operatorname{End}(B)}$. If $g$ does not split any element of $X$, then $g=g_{1} \times g_{2}$ for some $g_{1}, g_{2} \in S(X)$. Suppose that $g$ splits an element of $X$. Define $g_{1}, g_{2} \in S(X)$ by $i g_{1}=p_{i}$ and $i g_{2}=k_{i}$, where $p_{i}$ and $k_{i}$ are the elements from Lemma $4.2(i=1,2)$. It is routine to check that, for all $k, p \in X,(k, p) g=\left(p g_{2}, k g_{1}\right)$, that is, $g=\left(g_{1} \times g_{2}\right) \delta$.

We now consider $B=X \times X$ with $|X|>2$. Here we use the fact that $\operatorname{End}(B) \cong$ $T(X) \times T(X)$. Let $S$ be a characteristic subsemigroup of a semigroup $T$. Then a lift of $\phi \in \operatorname{Aut}(S)$ is any $\psi \in \operatorname{Aut}(T)$ such that $\psi \mid S=\phi$.

Lemma 4.4. Let $X$ be a set with $|X|>2, T=T(X)$, and $\psi: T \times T \rightarrow T \times T$. Then $\psi \in \operatorname{Aut}(T \times T)$ if and only if there exist $g_{1}, g_{2} \in S(X)$ such that $\psi=\phi_{T}^{g_{1}} \times \phi_{T}^{g_{2}}$ or $\psi=\left(\phi_{T}^{g_{1}} \times \phi_{T}^{g_{2}}\right) \lambda$, where $\lambda: T \times T \rightarrow T \times T$ is defined by $(a, b) \lambda=(b, a)$.

Proof. Let $S=S(X)$, and let $\phi: S \times S \rightarrow S \times S$. It is well known that if $|X|>2$, then $\phi \in \operatorname{Aut}(S \times S)$ if and only if there exist $\phi_{1}, \phi_{2} \in \operatorname{Aut}(S)$ such that $\phi=\phi_{1} \times \phi_{2}$ or $\phi=\left(\phi_{1} \times \phi_{2}\right) \mu$, where $\mu: S \times S \rightarrow S \times S$ is defined by $(g, h) \mu=(h, g)$.

Since $S \times S$ is the group of units of $T \times T$, it is characteristic in $T \times T$. Thus, the automorphisms of $T \times T$ are precisely the lifts of the automorphisms of $S \times S$.

We claim that every $\phi \in \operatorname{Aut}(S \times S)$ has at most one lift to $T \times T$, or equivalently, that $\operatorname{id}_{S \times S}$, the identity of $\operatorname{Aut}(S \times S)$, has exactly one lift, namely $\operatorname{id}_{T \times T}$. Indeed, suppose $\tau$ is a lift of $\operatorname{id}_{S \times S}$, that is, $\tau \in \operatorname{Aut}(T \times T)$ and $\tau \mid S \times S=\operatorname{id}_{S \times S}$. For $u, v \in T$, let 
$(u, v) \tau=\left(u^{*}, v^{*}\right)$. For $x \in X$, denote by $a_{x}$ the element of $T$ with range $\{x\}$, and by $S_{x}$ denote the stabilizer of $x$ in $S$. Let $u \in T$. Then $u g=u$ for every $g \in S_{x}$ if and only if $u=a_{x}$. Let $x, y \in X$. Then, for all $g \in S_{x}$ and $h \in S_{y}$,

$$
\left(a_{x}^{*}, a_{y}^{*}\right)=\left(a_{x}, a_{y}\right) \tau=\left(\left(a_{x}, a_{y}\right)(g, h)\right) \tau=\left(a_{x}, a_{y}\right) \tau(g, h) \tau=\left(a_{x}, a_{y}\right) \tau(g, h)=\left(a_{x}^{*} g, a_{y}^{*} h\right) .
$$

It follows that, for all $x, y \in X,\left(a_{x}^{*}, a_{y}^{*}\right)=\left(a_{x}, a_{y}\right)$, that is, $\left(a_{x}, a_{y}\right) \tau=\left(a_{x}, a_{y}\right)$.

Now let $x, y \in X$ and $u, v \in T$. It is obvious that $\left(a_{x}, a_{y}\right)(u, v)=\left(a_{x u}, a_{y v}\right)$. Thus,

$$
\begin{aligned}
\left(a_{x u}, a_{y v}\right) & =\left(a_{x u}, a_{y v}\right) \tau=\left(\left(a_{x}, a_{y}\right)(u, v)\right) \tau=\left(a_{x}, a_{y}\right) \tau(u, v) \tau \\
& =\left(a_{x}, a_{y}\right)((u, v) \tau)=\left(a_{x}, a_{y}\right)\left(u^{*}, v^{*}\right)=\left(a_{x u^{*}}, a_{y v^{*}}\right) .
\end{aligned}
$$

Thus, if $\left(u^{*}, v^{*}\right)=(u, v) \tau$, then $x u^{*}=x u$ and $y v^{*}=y v$ for all $x, y \in X$. It follows that $(u, v) \tau=(u, v)$, so $\tau=\mathrm{id}_{T \times T}$. The claim has been proved.

Now, let $\psi \in \operatorname{Aut}(T \times T)$. Then $\psi$ is a lift of $\phi=\psi \mid S \times S \in \operatorname{Aut}(S \times S)$. Since $|X|>2$, there exist $\phi_{1}, \phi_{2} \in \operatorname{Aut}(S)$ such that $\phi=\phi_{1} \times \phi_{2}$ or $\phi=\left(\phi_{1} \times \phi_{2}\right) \mu$. Suppose $|X| \neq 6$. Then every automorphism of $S=S(X)$ is inner, so $\phi_{1}=\phi_{S}^{g_{1}}$ and $\phi_{2}=\phi_{S}^{g_{2}}$ for some $g_{1}, g_{2} \in S(X)$. Then $\phi_{1} \times \phi_{2}$ can be lifted to $\phi_{T}^{g_{1}} \times \phi_{T}^{g_{2}} \in \operatorname{Aut}(T \times T)$. Clearly, $\mu$ can be lifted to $\lambda$. Since a lift of $\phi$ is unique, we have $\psi=\phi_{T}^{g_{1}} \times \phi_{T}^{g_{2}}$ or $\psi=\left(\phi_{T}^{g_{1}} \times \phi_{T}^{g_{2}}\right) \lambda$.

If $|X|=6$, then there are outer automorphisms of $S(X)$. However, we will prove that since $\phi$ can be lifted to $\psi, \phi_{1}$ and $\phi_{2}$ must be inner. Suppose $\phi=\phi_{1} \times \phi_{2}$. We claim that $(T \times\{1\}) \psi=T \times\{1\}$, where $1=\operatorname{id}_{X}$. For $x \in X$, let $\left(a_{x}, 1\right) \psi=\left(u_{x}, v_{x}\right)$. Let $g \in S$ and $x \in X$. Since $\left(a_{x}, 1\right)=(g, 1)\left(a_{x}, 1\right)$, we have

$$
\left(u_{x}, v_{x}\right)=(g, 1) \phi\left(a_{x}, 1\right) \psi=\left(g \phi_{1}, 1 \phi_{2}\right)\left(u_{x}, v_{x}\right)=\left(g \phi_{1} u_{x}, v_{x}\right) .
$$

Since $g$ was arbitrary, it follows that $u_{x}=h u_{x}$ for every $h \in G$, which implies that $u_{x}$ is a constant mapping. Thus, for every $x \in X,\left(a_{x}, 1\right) \psi=\left(a_{x_{1}}, v_{x}\right)$, where $x_{1} \in X$. In a similar way, we prove that, for every $y \in X,\left(1, a_{y}\right) \psi=\left(w_{y}, a_{y_{2}}\right)$, where $w_{y} \in T$ and $y_{2} \in X$.

It is clear that $M=\left\{\left(a_{x}, a_{y}\right): x, y \in X\right\}$ is the minimal ideal of $T \times T$. Let $x^{\prime}, y^{\prime} \in X$. Since $\psi$ maps $M$ onto $M$, there exist $x, y \in X$ such that $\left(a_{x}, a_{y}\right) \psi=\left(a_{x^{\prime}}, a_{y^{\prime}}\right)$. Since $\left(a_{x}, a_{y}\right)=\left(a_{x}, 1\right)\left(1, a_{y}\right)$, we have

$$
\left(a_{x^{\prime}}, a_{y^{\prime}}\right)=\left(a_{x}, 1\right) \psi\left(1, a_{y}\right) \psi=\left(a_{x_{1}}, v_{x}\right)\left(w_{y}, a_{y_{2}}\right)=\left(a_{x_{1}} w_{y}, v_{x} a_{y_{2}}\right) .
$$

Thus, $a_{y^{\prime}}=v_{x} a_{y_{2}}$, which implies $y^{\prime}=y_{2}$. Using $\left(a_{x}, a_{y}\right)=\left(1, a_{y}\right)\left(a_{x}, 1\right)$, we obtain $a_{y^{\prime}}=a_{y_{2}} v_{x}=a_{y^{\prime}} v_{x}$. This gives $y^{\prime} v_{x}=y^{\prime}$, and so, since $y^{\prime}$ was arbitrary, $v_{x}=1$. Let $z \in X$. Select $g \in S$ such that $x g=z$. Then $\left(a_{z}, 1\right)=\left(a_{x}, 1\right)(g, 1)$, and so

$$
\left(a_{z_{1}}, v_{z}\right)=\left(a_{x}, 1\right) \psi(g, 1) \phi=\left(a_{x_{1}}, 1\right)\left(g \phi_{1}, 1\right)=\left(a_{x_{1}} g \phi_{1}, 1\right) .
$$

Thus, $v_{z}=1$. We proved that, for every $x \in X,\left(a_{x}, 1\right) \psi=\left(a_{x_{1}}, 1\right)$.

Let $a \in T$ and $x \in X$. Let $(a, 1) \psi=\left(u_{a}, v_{a}\right)$. Since $\left(a_{x}, 1\right)=(a, 1)\left(a_{x}, 1\right)$, we have

$$
\left(a_{x_{1}}, 1\right)=(a, 1) \psi\left(a_{x}, 1\right) \psi=\left(u_{a}, v_{a}\right)\left(a_{x_{1}}, 1\right)=\left(a_{x_{1}}, v_{a}\right) .
$$

Thus, $v_{a}=1$, and so $(a, 1) \psi=\left(u_{a}, 1\right)$. The claim has been proved. 
Define $\psi_{1}: T \rightarrow T$ by $a \psi_{1}=u_{a}$. It is clear that $\psi_{1} \in \operatorname{Aut}(T)$ and $\psi_{1} \mid S=\phi_{1}$. It is well known that all automorphisms of $T(X)$ are inner. Thus, $\psi_{1}$ is inner, and so $\phi_{1}$ is also inner. In a similar way, we prove that $(\{1\} \times T) \psi=\{1\} \times T$, which implies that $\phi_{2}$ is inner. A proof that $\phi_{1}$ and $\phi_{2}$ are inner when $\phi=\left(\phi_{1} \times \phi_{2}\right) \mu$ is similar. (In this case, we have $(T \times\{1\}) \psi=\{1\} \times T$ and $(\{1\} \times T) \psi=T \times\{1\}$.) Now we complete the proof as in the case when $|X| \neq 6$.

Let $C_{2}=\{1, t\}$ be the cyclic group of order 2 . Consider the semidirect product $[S(X) \times$ $S(X)] \ltimes C_{2}$, where $C_{2}$ acts on $S(X) \times S(X)$ by $\left(g_{1}, g_{2}\right)^{1}=\left(g_{1}, g_{2}\right)$ and $\left(g_{1}, g_{2}\right)^{t}=\left(g_{2}, g_{1}\right)$. Note that the multiplication in $[S(X) \times S(X)] \ltimes C_{2}$ is given by

$$
\begin{aligned}
& \left(\left(g_{1}, g_{2}\right), 1\right)\left(\left(h_{1}, h_{2}\right), s\right)=\left(\left(g_{1}, g_{2}\right)\left(h_{1}, h_{2}\right)^{1}, 1 s\right)=\left(\left(g_{1} h_{1}, g_{2} h_{2}\right), s\right), \\
& \left(\left(g_{1}, g_{2}\right), t\right)\left(\left(h_{1}, h_{2}\right), s\right)=\left(\left(g_{1}, g_{2}\right)\left(h_{1}, h_{2}\right)^{t}, t s\right)=\left(\left(g_{1} h_{2}, g_{2} h_{1}\right), t s\right) .
\end{aligned}
$$

Let $M=\{1,2\}$. We can interpret $S(X) \times S(X)$ as $S(X)^{M}$ (the set of functions from $M$ to $S(X))$ and $C_{2}$ as $S(M)$. With this interpretation, the semidirect product $[S(X) \times$ $S(X)] \ltimes C_{2}$ becomes the wreath product $S(X) \prec C_{2}$.

Theorem 4.5. Let $X$ be a non-empty set, let $\mathcal{V}$ be a variety of rectangular bands and let $B=B_{\mathcal{V}}(X)$. Then we have the following.

(1) If $\mathcal{V}=\mathcal{L} \mathcal{Z}$ or $\mathcal{V}=\mathcal{R Z}$, then $\operatorname{Aut}(\operatorname{End}(B)) \cong S(X)$.

(2) If $\mathcal{V}=\mathcal{R B}$ and $|X| \geqslant 2$, then $\operatorname{Aut}(\operatorname{End}(B)) \cong S(X) \prec C_{2}$.

Proof. We established (1) at the beginning of this section. To prove (2), let $B$ be a free rectangular band. By Lemma 4.1, $B=X \times X$.

Suppose that $|X|=2$ and let $g \in G_{\operatorname{End}(B)}$. By Lemma $4.3, g=g_{1} \times g_{2}$ or $g=\left(g_{1} \times g_{2}\right) \delta$, where $g_{1}, g_{2} \in S(X)$ and $(k, p) \delta=(p, k)(k, p \in X)$. Map $G_{\operatorname{End}(B)}$ to $[S(X) \times S(X)] \ltimes C_{2}$ by

$$
g_{1} \times g_{2} \rightarrow\left(\left(g_{1}, g_{2}\right), 1\right) \quad \text { and } \quad\left(g_{1} \times g_{2}\right) \delta \rightarrow\left(\left(g_{1}, g_{2}\right), t\right) .
$$

It is routine to check that this mapping is a group isomorphism. Thus, $\operatorname{Aut}(\operatorname{End}(B)) \cong$ $G_{\operatorname{End}(B)} \cong[S(X) \times S(X)] \ltimes C_{2} \cong S(X)<C_{2}$.

Suppose that $|X|>2$ and let $\psi \in \operatorname{Aut}(T(X) \times T(X))$ and $T=T(X)$. By Lemma 4.4, $\psi=\phi_{T}^{g_{1}} \times \phi_{T}^{g_{1}}$ or $\psi=\left(\phi_{T}^{g_{1}} \times \phi_{T}^{g_{1}}\right) \lambda$, where $g_{1}, g_{2} \in S(X)$ and $(a, b) \lambda=(b, a)(a, b \in$ $T(X))$. Map $\operatorname{Aut}(T(X) \times T(X))$ to $[S(X) \times S(X)] \ltimes C_{2}$ by

$$
\phi_{T}^{g_{1}} \times \phi_{T}^{g_{1}} \rightarrow\left(\left(g_{1}, g_{2}\right), 1\right) \quad \text { and } \quad\left(\phi_{T}^{g_{1}} \times \phi_{T}^{g_{1}}\right) \lambda \rightarrow\left(\left(g_{1}, g_{2}\right), t\right) .
$$

Again, it is routine to check that this mapping is an isomorphism. Thus,

$$
\operatorname{Aut}(\operatorname{End}(B)) \cong \operatorname{Aut}(T(X) \times T(X)) \cong[S(X) \times S(X)] \ltimes C_{2} \cong S(X) \prec C_{2}
$$




\section{Relatively free bands that are $d$-invariant}

Let $\mathcal{V}$ be a variety of bands with $\mathcal{S L} \subseteq \mathcal{V}$ and $\mathcal{S L} \neq \mathcal{V}$. Then, by Theorem 3.8, the group $\operatorname{Aut}\left(\operatorname{End}\left(B_{\mathcal{V}}(X)\right)\right)$ is isomorphic either to $S(X) \times C_{2}$ (if $B_{\mathcal{V}}(X)$ is $d$-invariant) or to $S(X)$ (if $B_{\mathcal{V}}(X)$ is not $d$-invariant). Therefore, to have a complete picture, we need to determine which relatively free bands $B_{\mathcal{V}}(X)$ are $d$-invariant and which are not.

Figure 1 shows the lattice of band varieties. At the bottom we have the sublattice generated by the atoms $\mathcal{S} \mathcal{L}=[x y=y x], \mathcal{L Z}=[x y=x]$ and $\mathcal{R Z}=[x y=y]$ (see $[\mathbf{1 3}$, p. 137]). In addition to the atoms, the sublattice contains the varieties $\mathcal{T}=[x=y]$ of trivial bands, $\mathcal{R B}=[x y x=x]$ of rectangular bands, $\mathcal{L N}=[x y z=x z y]$ of left normal bands, $\mathcal{R N}=[x y z=y x z]$ of right normal bands and $\mathcal{N}=[x y z x=x z y x]$ of normal bands. (Note that we omit the identity $x^{2}=x$ in the system of identities that define band varieties.) At the top we have the variety $\mathcal{B}$ of all bands. The remaining varieties are labelled with the identities given in [10]. The words that occur in the identities are defined inductively as follows:

$$
\begin{array}{llll}
G_{2} & =x_{2} x_{1}, & H_{2}=x_{2}, & I_{2}=x_{2} x_{1} x_{2}, \\
G_{n}=x_{n} \bar{G}_{n-1}, & H_{n}=G_{n} x_{n} \bar{H}_{n-1}, & I_{n}=G_{n} x_{n} \bar{I}_{n-1}, \quad(n \geqslant 3) .
\end{array}
$$

For example, $G_{3}=x_{3} x_{1} x_{2}$ and $H_{3}=x_{3} x_{1} x_{2} x_{3} x_{2}$. Thus, if $\mathcal{V}$ is the variety labelled $G_{3}=H_{3}$ (second from the bottom in column 1), then $\mathcal{V}=\left[x_{3} x_{1} x_{2}=x_{3} x_{1} x_{2} x_{3} x_{2}\right]$.

The varieties $\mathcal{V}$ in column 3 are self-dual, that is, if $\mathcal{V}$ is given by the identity $A=B$, then $\bar{A}=A$ and $\bar{B}=B$. It easily follows that, for all $u, v \in B_{\mathcal{V}}(X)$,

$$
u=v \text { in } B_{\mathcal{V}}(X) \Longleftrightarrow \bar{u}=\bar{v} \text { in } B_{\mathcal{V}}(X) .
$$

In other words, for every variety $\mathcal{V}$ in column 3 and every set $X, B_{\mathcal{V}}(X)$ is $d$-invariant.

The varieties in column 5 are dual to the corresponding (same-level) varieties in column 1 . That is, if a variety $\mathcal{V}$ in column 1 is given by the identity $A=B$, then the corresponding variety $\mathcal{V}^{*}$ in column 5 is given by $\bar{A}=\bar{B}$. Similarly, the varieties in column 4 are dual to the corresponding varieties in column 2 .

Let $\mathcal{V}=[A=B]$ be a variety in column $1($ column 2$)$ and $\mathcal{V}^{*}=[\bar{A}=\bar{B}]$ the dual variety in column 5 (column 4 ). Let $X$ be any set with cardinality at least $\max \{|C(A)|,|C(B)|\}$. Then $\bar{A} \neq \bar{B}$ in $B \mathcal{V}(X)$, since otherwise every semigroup $S$ in $\mathcal{V}$ would satisfy the identity $\bar{A}=\bar{B}$ and we would have $\mathcal{V} \subseteq \mathcal{V}^{*}$, which is a contradiction. Hence, for every variety $\mathcal{V}=[A=B]$ not in column 3 and every set $X$ with $|X| \geqslant n$, where $n$ is the number of variables (letters) in the identity $A=B, B_{\mathcal{V}}(X)$ is not $d$-invariant.

The foregoing discussion and Theorems 3.8 and 4.5 give us the following corollaries.

Corollary 5.1. Let $X$ be any set with $|X| \geqslant 2$, let $\mathcal{V}$ be any variety of bands in column 3 of Figure 1 (including $\mathcal{B}$ ), and let $B=B_{\mathcal{V}}(X)$. Then we have the following.

(1) If $\mathcal{V}=\mathcal{T}$, then $\operatorname{Aut}(\operatorname{End}(B))=\{1\}$.

(2) If $\mathcal{V}=\mathcal{S} \mathcal{L}$, then $\operatorname{Aut}(\operatorname{End}(B)) \cong S(X)$.

(3) If $\mathcal{V}=\mathcal{R B}$, then $\operatorname{Aut}(\operatorname{End}(B)) \cong S(X) \curlywedge C_{2}$.

(4) If $\mathcal{V} \neq \mathcal{T}, \mathcal{V} \neq \mathcal{S} \mathcal{L}$, and $\mathcal{V} \neq \mathcal{R B}$, then $\operatorname{Aut}(\operatorname{End}(B)) \cong S(X) \times C_{2}$. 
$\mathcal{B}$

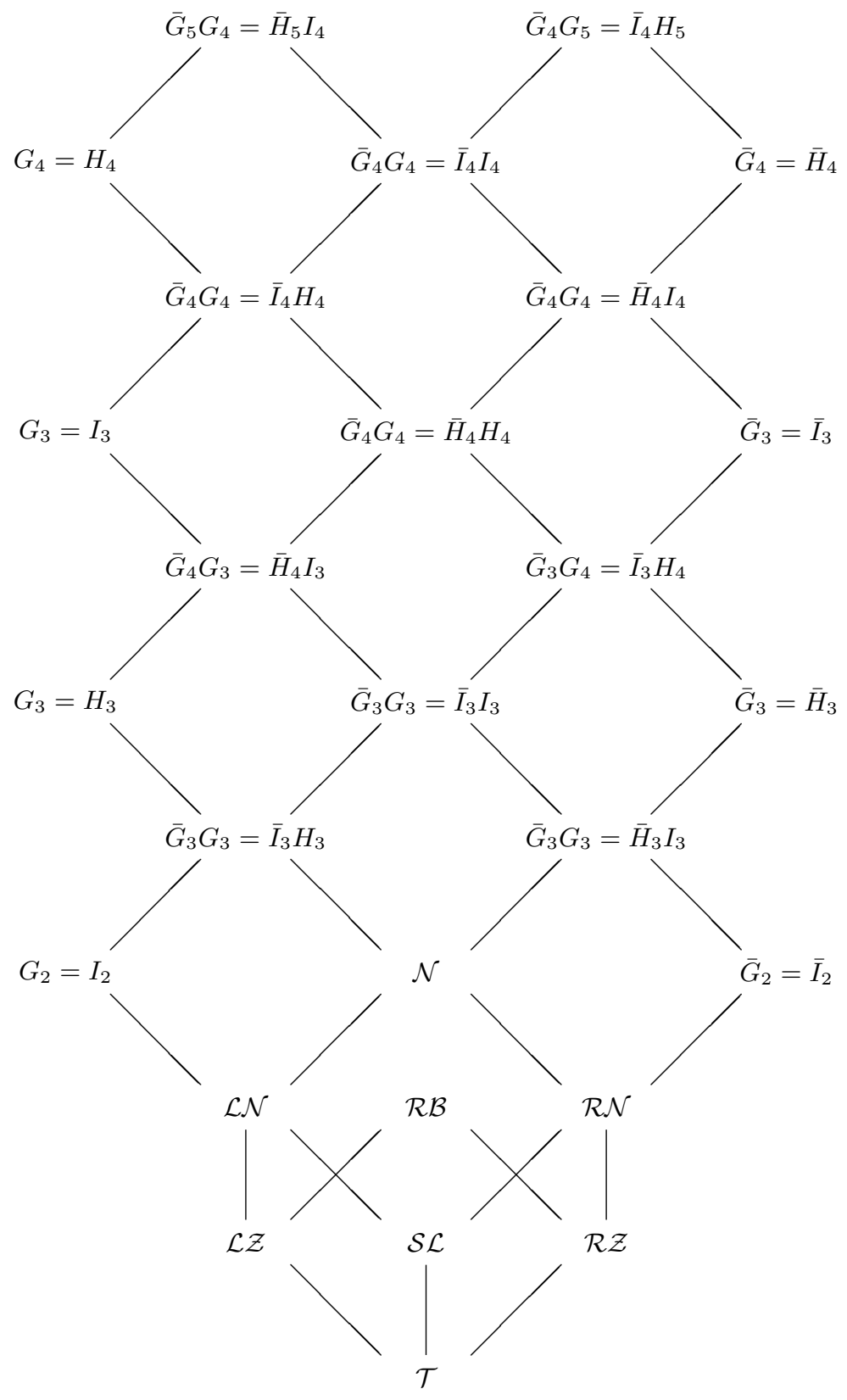

Figure 1. The lattice of band varieties. 
Corollary 5.2. Let $\mathcal{V}=[A=B]$ be any variety of bands in Figure 1 that is not in column 3. Then, for every set $X$ with $|X| \geqslant n$, where $n$ is the number of variables in $A=B$,

$$
\operatorname{Aut}\left(\operatorname{End}\left(B_{\mathcal{V}}(X)\right)\right) \cong S(X) .
$$

It remains to investigate the free bands $B_{\mathcal{V}}(X)$ such that $\mathcal{V}=[A=B]$ is not in column 3 and $|X|<n$, where $n$ is the number of variables in $A=B$. By duality, it suffices to consider the varieties in columns 1 and 2 .

We will use the operators on $X^{*}=X^{+} \cup\{1\}$ introduced in [10], which solve the word problem in the relatively free bands (see also $[\mathbf{2 0}, \mathbf{2 3}]$ ). We say that an operator $t: X^{*} \rightarrow$ $X^{*}$ solves the word problem in the relatively free band $B_{\mathcal{V}}(X)$ if for all $u, v \in X^{+}, u=v$ in $B_{\mathcal{V}}(X)$ if and only if $t(u)=t(v)$ in $X^{*}$. (Note that we write the operators on $X^{*}$ on the left, that is, $t(w)$ rather than $w t$.) Any such operator $t$ is called an invariant for the variety $\mathcal{V}$. (We assume, of course, that the definition of $t$ makes sense for every non-empty set $X$.)

Following [10], we define operators $h_{2}, i_{2}, \sigma, s, \varepsilon$ and $e$ on $X^{*}$ by $h_{2}(1)=i_{2}(1)=$ $\sigma(1)=s(1)=\varepsilon(1)=e(1)=1$ and, for any $w \in X^{+}$,

$h_{2}(w)=$ the first letter occurring in $w$,

$i_{2}(w)=$ the word obtained from $w$ by retaining only the first occurrence of each letter,

$\sigma(w)=$ the letter in $w$ that is last to make its first appearance,

$s(w)=$ the subword of $w$ that precedes the first appearance of $\sigma(w)$,

$\varepsilon(w)=$ the letter in $w$ that is first to make its last appearance,

$e(w)=$ the subword of $w$ that follows the last appearance of $\varepsilon(w)$.

For example, $h_{2}(x z x y z)=x, i_{2}(x z x y z)=x z y, \sigma(x z x y z)=y, s(x z x y z)=x z x$, $\varepsilon(x z x y z)=x$ and $e(x z x y x)=y z$.

For an operator $t: X^{*} \rightarrow X^{*}$, define the dual operator $\bar{t}$ by $\bar{t}(w)=\overline{t(\bar{w})}$. For $n \geqslant 3$, define inductively operators $h_{n}$ and $i_{n}$ on $X^{*}$ by

$$
\begin{aligned}
h_{n}(w) & =h_{n}(s(w)) \sigma(w) \bar{h}_{n-1}(w), \\
i_{n}(w) & =i_{n}(s(w)) \sigma(w) \bar{i}_{n-1}(w) .
\end{aligned}
$$

The operators $h_{n}(n \geqslant 3)$ and $i_{n}(n \geqslant 2)$ are invariants for the varieties $\left[G_{n}=H_{n}\right]$ and $\left[G_{n}=I_{n}\right]$, respectively [10]. The duals $\bar{h}_{n}$ and $\bar{i}_{n}$ are invariants for the corresponding dual varieties. The operators $h_{2}$ and $\bar{h}_{2}$ are invariants for $\mathcal{L Z}$ and $\mathcal{R Z}$, respectively. Recall that, for the variety $\mathcal{S L}$ of semilattices, we have $u=v$ in $B_{\mathcal{S L}}(X)$ if and only if $C(u)=C(v)$. If $t_{1}$ and $t_{2}$ are invariants for varieties $\mathcal{V}_{1}$ and $\mathcal{V}_{2}$, respectively, then the conjunction of $t_{1}$ and $t_{2}$ is an invariant for the join $\mathcal{V}_{1} \vee \mathcal{V}_{2}$. For example, since $\mathcal{V}=\left[\bar{G}_{4} G_{3}=\bar{H}_{4} I_{3}\right]$ is the join of $\left[G_{3}=H_{3}\right]$ and $\left[\bar{G}_{2}=\bar{I}_{2}\right]$ (see Figure 1), then, for all $u, v \in X^{+}, u=v$ in $B_{\mathcal{V}}(X)$ if and only if $h_{3}(u)=h_{3}(v)$ and $\bar{i}_{2}(u)=\bar{i}_{2}(v)$ in $X^{+}$.

The above paragraph gives us invariants for all band varieties except the variety $\mathcal{B}$ of all bands. An invariant $b: X^{*} \rightarrow X^{*}$ for $\mathcal{B}$ is given in [10]. We define $b(w)$ inductively 
on the number of letters in a word $w$ as follows:

$$
b(1)=1 \quad \text { and } \quad b(w)=b(s(w)) \sigma(w) \varepsilon(w) b(e(w)), \quad w \in X^{+} .
$$

\subsection{Column 1}

We show that, for all varieties $\mathcal{V}=[A=B]$ in column 1 , if $|X|<n$, where $n$ is the number of variables in $A=B$, then $B_{\mathcal{V}}(X)$ is $d$-invariant. The result will follow from the following lemma.

Lemma 5.3. Let $X$ be a non-empty set and $n \geqslant 3$. For all words $u, v \in X^{+}$, if $u=v$ has less than $n$ variables and $h_{n}(u)=h_{n}(v)$, then $b(u)=b(v)$.

Proof. We proceed by induction on $n$. Let $n=3$. By [23, Proposition 3.7], $h_{3}(u)=$ $h_{3}(v)$ implies $h_{3}(s(u))=h_{3}(s(v)), \sigma(u)=\sigma(v)$, and $\bar{h}_{2}(u)=\bar{h}_{2}(v)$. Since $n=3, u=v$ has one or two variables. If $u=v$ has one variable, the result is trivial since $b(u)=b(v)$ for all words $u$ and $v$ in a single variable $x$. Suppose $u=v$ has two variables, say $x$ and $y$. Then $s(u)=s(v)$ has one variable, and $b(s(u))=b(s(v))$ as above. We have $\bar{h}_{2}(u)=\bar{h}_{2}(v)$, that is, $u$ and $v$ have the same last variable, say $y$. It follows that $\varepsilon(u)=\varepsilon(v)=x$. Thus, $e(u)=e(v)$ has a single variable, $y$, and so $b(e(u))=b(e(v))$. Hence, $b(u)=b(v)$.

Let $n \geqslant 4$. By [23, Proposition 3.4], for every word $w$,

$$
h_{n}(w)=h_{n}(s(w)) \sigma(w) \bar{h}_{n-1}(w)=h_{n}(s(w)) \sigma(w) h_{n-2}(w) \varepsilon(w) \bar{h}_{n-1}(e(w)) .
$$

Again by [23, Proposition 3.7], $h_{n}(u)=h_{n}(v)$ implies that $h_{n}(s(u))=h_{n}(s(v)), \sigma(u)=$ $\sigma(v), \varepsilon(u)=\varepsilon(v)$ and $\bar{h}_{n-1}(e(u))=\bar{h}_{n-1}(e(v))$. Furthermore, $\bar{h}_{n-1}(e(u))=\bar{h}_{n-1}(e(v))$ implies that $h_{n-1}(\overline{e(u)})=h_{n-1}(\overline{e(v)})$. Thus, since $e(u)=e(v)$ has at most $n-2$ variables, $b(\overline{e(u)})=b(\overline{e(v)})$ by induction. Thus, $b(e(u))=b(e(v))$ since the variety $\mathcal{B}$ of all bands is self-dual.

To complete the proof that $b(u)=b(v)$, we use the induction on the number of variables in $u=v$. If $u=v$ has one variable, the result is trivial. Suppose $u=v$ has at least two variables. Then $h_{n}(s(u))=h_{n}(s(v))$ implies that $b(s(u))=b(s(v))$, since $s(u)=s(v)$ has one less variable than $u=v$. We already established $\sigma(u)=\sigma(v), \varepsilon(u)=\varepsilon(v)$ and $b(e(u))=b(e(v))$. It follows that $b(u)=b(v)$.

Proposition 5.4. Let $\mathcal{V}=[A=B]$ be any variety of bands in column 1 or column 5 of Figure 1. Then, for every set $X$ with $|X|<n$, where $n$ is the number of variables in $A=B, B_{\mathcal{V}}(X)$ is d-invariant.

Proof. By duality, we may assume that $\mathcal{V}$ is in column 1 . Suppose that $\mathcal{V}=\left[G_{n}=\right.$ $\left.H_{n}\right], n \geqslant 3$. Let $u, v \in X^{+}$be such that $u=v$ in $B_{\mathcal{V}}(X)$, that is, $h_{n}(u)=h_{n}(v)$. Since $|X|<n$, Lemma 5.3 gives $b(u)=b(v)$, that is, $u=v$ in $B_{\mathcal{B}}(X)$, where $\mathcal{B}$ is the variety of all bands. Since $B_{\mathcal{B}}(X)$ is $d$-invariant, $\bar{u}=\bar{v}$ in $B_{\mathcal{B}}(X)$, and so $\bar{u}=\bar{v}$ in $B_{\mathcal{V}}(X)$ since $\mathcal{V} \subset \mathcal{B}$. 
Suppose $\mathcal{V}=\left[G_{n}=I_{n}\right], n \geqslant 2$. If $n=2$, the result is trivial. Let $n \geqslant 3$. Then $\mathcal{V}_{1}=\left[G_{n}=H_{n}\right]$ is included in $\mathcal{V}$ (see Figure 1). Thus, for all $u, v \in X^{+}$,

$$
\begin{aligned}
& u=v \text { in } B_{\mathcal{V}}(X) \quad \Longrightarrow \quad u=v \text { in } B_{\mathcal{V}_{1}}(X) \\
& \Longrightarrow u=v \text { in } B_{\mathcal{B}}(X) \\
& \Longrightarrow \quad \bar{u}=\bar{v} \text { in } B_{\mathcal{B}}(X) \\
& \Longrightarrow \quad \bar{u}=\bar{v} \text { in } B_{\mathcal{V}}(X) \text {. }
\end{aligned}
$$

This concludes the proof.

Proposition 5.4, together with Theorem 3.8, gives us the following corollary.

Corollary 5.5. Let $\mathcal{V}=[A=B]$ be any variety of bands in column 1 or column 5 of Figure 1. Then for every set $X$ with $2 \leqslant|X|<n$, where $n$ is the number of variables in $A=B, \operatorname{Aut}\left(\operatorname{End}\left(B_{\mathcal{V}}(X)\right)\right) \cong S(X) \times C_{2}$.

\subsection{Column 2}

Here the situation is more complicated than it is in column 1. Proposition 5.4 is true for the varieties $\mathcal{V}=\left[\bar{G}_{n} G_{n}=\bar{I}_{n} H_{n}\right], n \geqslant 3$, and the variety $\mathcal{L} \mathcal{Z}$ of left zero semigroups, but not for the varieties $\mathcal{V}=\left[\bar{G}_{n} G_{n-1}=\bar{H}_{n} I_{n-1}\right], n \geqslant 3$, or the variety $\mathcal{L N}$ of left normal bands.

We first consider the former, for which the result will follow from the following lemma, which is similar to Lemma 5.3.

Lemma 5.6. Let $X$ be a non-empty set and $n \geqslant 2$. For all words $u, v \in X^{+}$, if $u=v$ has at most $n$ variables, $i_{n}(u)=i_{n}(v)$ and $\bar{h}_{n}(u)=\bar{h}_{n}(v)$, then $b(u)=b(v)$.

Proof. We proceed by induction on $n$. Let $n=2$. Then $u=v$ has one or two variables. If $u=v$ has one variable, the result is trivial. Suppose $u=v$ has two variables. Then $i_{2}(u)=i_{2}(v)$ implies that $\sigma(u)=\sigma(v)$ and that $s(u)=s(v)$ has one variable. Further, $\bar{h}_{2}(u)=\bar{h}_{2}(v)$ implies that $\varepsilon(u)=\varepsilon(v)$ and that $e(u)=e(v)$ has one variable. It follows that $b(u)=b(v)$.

Let $n \geqslant 3$. Since $\left[G_{n}=H_{n}\right] \subset\left[G_{n}=I_{n}\right]$ (see Figure 1), $i_{n}(u)=i_{n}(v)$ implies $h_{n}(u)=h_{n}(v)$. If $u=v$ has less than $n$ variables, then $h_{n}(u)=h_{n}(v)$ implies $b(u)=b(v)$ by Lemma 5.3. Suppose $u=v$ has $n$ variables. We have that $i_{n}(u)=i_{n}(v)$ implies $i_{n}(s(u))=i_{n}(s(v)), \sigma(u)=\sigma(v)$ and $\bar{i}_{n-1}(u)=\bar{i}_{n-1}(v)$, and $\bar{h}_{n}(u)=\bar{h}_{n}(v)$ implies that $h_{n-1}(u)=h_{n-1}(v), \varepsilon(u)=\varepsilon(v)$ and $\bar{h}_{n}(e(u))=\bar{h}_{n}(e(v))$ (by [23, Proposition 3.7]). Since $s(u)=s(v)$ and $e(u)=e(v)$ have $n-1$ variables each, it follows by Lemma 5.3 that $b(s(u))=b(s(v))$ and $b(e(u))=b(e(v))$. Thus, $b(u)=b(v)$.

Proposition 5.7. Let $\mathcal{V}$ be a variety of bands in column 2 or column 4 of Figure 1. If $\mathcal{V}=\left[\bar{G}_{n} G_{n}=\bar{I}_{n} H_{n}\right]$ or $\mathcal{V}=\left[\bar{G}_{n} G_{n}=\bar{H}_{n} I_{n}\right], n \geqslant 3$, then, for every set $X$ with $|X|<n$, $B_{\mathcal{V}}(X)$ is d-invariant. 
Proof. By duality, we may assume that $\mathcal{V}=\left[\bar{G}_{n} G_{n}=\bar{I}_{n} H_{n}\right]$. Then for all $u, v \in X^{+}$, $u=v$ in $B \mathcal{V}(X)$ if and only if $i_{n-1}(u)=i_{n-1}(v)$ and $\bar{h}_{n-1}(u)=\bar{h}_{n-1}(v)$. Thus, the result follows from Lemma 5.6 .

For the varieties $\mathcal{V}=\left[\bar{G}_{n} G_{n-1}=\bar{H}_{n} I_{n-1}\right]$ and their duals, the result is as follows.

Proposition 5.8. Let $\mathcal{V}$ be a variety of bands in column 2 or column 4 of Figure 1. If $\mathcal{V}=\left[\bar{G}_{n} G_{n-1}=\bar{H}_{n} I_{n-1}\right]$ or $\mathcal{V}=\left[\bar{G}_{n-1} G_{n}=\bar{I}_{n-1} H_{n}\right], n \geqslant 4$, then, for every set $X$,

(1) if $|X|<n-1$, then $B_{\mathcal{V}}(X)$ is d-invariant,

(2) if $|X|=n-1$, then $B_{\mathcal{V}}(X)$ is not d-invariant.

Proof. By duality, we may assume that $\mathcal{V}=\left[\bar{G}_{n} G_{n-1}=\bar{H}_{n} I_{n-1}\right]$. To prove (1), suppose $|X|<n-1$, and let $u, v \in X^{+}$with $u=v$ in $B_{\mathcal{V}}(X)$. Consider $\mathcal{V}_{1}=\left[G_{n-1}=H_{n-1}\right]$. Since $\mathcal{V}_{1} \subset \mathcal{V}, u=v$ in $B_{\mathcal{V}_{1}}(X)$, so $h_{n-1}(u)=h_{n-1}(v)$. Since $u=v$ has less than $n-1$ variables, $u=v$ in $B_{\mathcal{B}}(X)$ by Lemma 5.3. Thus, $\bar{u}=\bar{v}$ in $B_{\mathcal{B}}(X)$, and so $\bar{u}=\bar{v}$ in $B_{\mathcal{V}}(X)$.

To prove (2), suppose that $|X|=n-1$. Then $G_{n-1}, I_{n-1} \in X^{+}$. We have $G_{n-1}=I_{n-1}$ in $B_{\mathcal{V}}(X)$ (since $\mathcal{V} \subset\left[G_{n-1}=I_{n-1}\right]$ ) and $\bar{G}_{n-1} \neq \bar{I}_{n-1}$ in $B_{\mathcal{V}}(X)$ (since $\mathcal{V} \not \subset\left[\bar{G}_{n-1}=\right.$ $\left.\bar{I}_{n-1}\right]$ ) (see Figure 1 ). Thus, $B_{\mathcal{V}}(X)$ is not $d$-invariant.

We dealt with the variety $\mathcal{L} \mathcal{Z}=[x y=x]$ of left zero semigroups and its dual $\mathcal{R Z}=$ $[x y=y]$ in $\S 4$. It remains to consider the variety $\mathcal{L N}=[x y z=x z y]$ of left normal bands. (Its dual is the variety $\mathcal{R N}=[x y z=y x z]$ of right normal bands.) The identity $[x y z=x z y]$ defining $\mathcal{L N}$ has three variables. Let $X$ be a set with two variables, say $X=\{x, y\}$. Since $\mathcal{L N}$ is the join of $\mathcal{L Z}$ and $\mathcal{S} \mathcal{L}$, for all $u, v \in X^{+}, u=v$ in $B_{\mathcal{L N}}(X)$ if and only if $h_{2}(u)=h_{2}(v)$ and $C(u)=C(v)$. It follows that $B_{\mathcal{L N}}(X)$ is not $d$-invariant, since $x y=x y x$ in $B_{\mathcal{L N}}(X)$ but $y x \neq x y x$ in $B_{\mathcal{L N}}(X)$.

The foregoing discussion, Propositions 5.7 and 5.8 and Theorem 3.8 give us the following corollary.

Corollary 5.9. Let $\mathcal{V}$ be a variety of bands in column 2 or column 4 of Figure 1. Then, for every set $X$ with $|X| \geqslant 2$,

(1) if $\mathcal{V}=\left[\bar{G}_{n} G_{n}=\bar{I}_{n} H_{n}\right]$ or $\mathcal{V}=\left[\bar{G}_{n} G_{n}=\bar{H}_{n} I_{n}\right], n \geqslant 3$, and $|X|<n$, then $\operatorname{Aut}\left(\operatorname{End}\left(B_{\mathcal{V}}(X)\right)\right) \cong S(X) \times C_{2}$,

(2) if $\mathcal{V}=\left[\bar{G}_{n} G_{n-1}=\bar{H}_{n} I_{n-1}\right]$ or $\mathcal{V}=\left[\bar{G}_{n-1} G_{n}=\bar{I}_{n-1} H_{n}\right], n \geqslant 4$, and $|X|<n-1$, then $\operatorname{Aut}\left(\operatorname{End}\left(B_{\mathcal{V}}(X)\right)\right) \cong S(X) \times C_{2}$,

(3) if $\mathcal{V}=\left[\bar{G}_{n} G_{n-1}=\bar{H}_{n} I_{n-1}\right]$ or $\mathcal{V}=\left[\bar{G}_{n-1} G_{n}=\bar{I}_{n-1} H_{n}\right], n \geqslant 4$, and $|X|=n-1$, then $\operatorname{Aut}\left(\operatorname{End}\left(B_{\mathcal{V}}(X)\right)\right) \cong S(X)$,

(4) if $\mathcal{V}=\mathcal{L N}$ or $\mathcal{V}=\mathcal{R N}$, and $|X|=2$, then $\operatorname{Aut}\left(\operatorname{End}\left(B_{\mathcal{V}}(X)\right)\right) \cong S(X)$.

If $|X|=1$, say $X=\{x\}$, then for, every variety $\mathcal{V}$ of bands $B_{\mathcal{V}}(X)=\{x\}$, and so $\operatorname{Aut}\left(\operatorname{End}\left(B_{\mathcal{V}}(X)\right)\right)=\{1\}$. Hence, we have covered all possible varieties of bands $\mathcal{V}$ and all possible sets $X$, and our determination of $\operatorname{Aut}\left(\operatorname{End}\left(B_{\mathcal{V}}(X)\right)\right)$ is complete. 


\section{Problems}

In connection with the general problem that motivated this paper and in parallel with many similar open problems in various branches of algebra, we suggest the following problems from semigroup theory.

(1) Describe $\operatorname{Aut}(\operatorname{Aut}(F))$ and $\operatorname{Aut}(\operatorname{End}(F))$, where $F$ is a free inverse semigroup, that is, a free algebra in the variety of all inverse semigroups. (This is the most important free semigroup apart from the free group (see [18, Chapter VIII]).)

(2) Solve analogous problems for other varieties of inverse semigroups (see [18, Chapter $\mathrm{XII}]$ ).

(3) Describe $\operatorname{Aut}(\operatorname{Aut}(F))$ and $\operatorname{Aut}(\operatorname{End}(F))$, where $F$ is a free completely regular semigroup or a free $*$-regular semigroup (see $[\mathbf{1 4}, \mathbf{2 1}]$ ).

(4) For a pseudovariety $\mathcal{V}$ of semigroups, describe $\operatorname{Aut}(\operatorname{Aut}(F))$ and $\operatorname{Aut}(\operatorname{End}(F))$, where $F$ is the free pro- $\mathcal{V}$ semigroup over a finite alphabet (see $[\mathbf{1}, \mathbf{2}])$.

(5) Describe $\operatorname{Aut}(\operatorname{Aut}(F))$ and $\operatorname{Aut}(\operatorname{End}(F))$, where $F$ is an independence algebra (see $[5,12])$.

Acknowledgements. The authors thank Professor Arthur Gerhard for providing the statement and proof of Lemma 5.3. J.A. acknowledges the support of FCT and FEDER, Projecto 863 POCTI/143.

\section{References}

1. J. Almeida, Finite semigroups and universal algebra, Series in Algebra, Volume 3 (World Scientific River Edge, NJ, 1994).

2. J. Almeida AND P. Weil, Relatively free profinite monoids: an introduction and examples, in Semigroups, formal languages and groups (ed. J. Fountain and V. Gould), NATO Science Series C, Volume 466, pp. 73-117 (Kluwer, Dordrecht, 1995).

3. J. ARAÚJo AND J. KonieCzNY, Dense relations are determined by their endomorphism monoids, Semigroup Forum 70 (2005), 302-306.

4. A. P. Biruukov, Varieties of idempotent semigroups, Alg. Logika 9 (1970), 255-273 (in Russian).

5. P. J. Cameron and C. Szabó, Independence algebras, J. Lond. Math. Soc. 61 (2000), 321-334.

6. C. F. Fennemore, All varieties of bands, I, Math. Nachr. 48 (1971), 237-252.

7. C. F. Fennemore, All varieties of bands, II, Math. Nachr. 48 (1971), 253-262.

8. E. Formanek, A question of B. Plotkin about the semigroup of endomorphisms of a free group, Proc. Am. Math. Soc. 130 (2002), 935-937.

9. J. A. Gerhard, The lattice of equational classes of idempotent semigroups, J. Alg. 15 (1970) 195-224.

10. J. A. Gerhard and M. Petrich, Varieties of bands revisited, Proc. Lond. Math. Soc. 58 (1989), 323-350.

11. L. M. GLUSKǏN, Semigroups and rings of endomorphisms of linear spaces, I, Am. Math. Soc. Transl. 45 (1965), 105-137.

12. V. Gould, Independence algebras, Alg. Univers. 33 (1995), 294-318. 
13. J. M. HowIE, Fundamentals of semigroup theory (Oxford University Press, 1995).

14. J. KAĎOUREK AND L. PolÁk, On the word problem for free completely regular semigroups, Semigroup Forum 34 (1986), 127-138.

15. A. I. MaL'Cev, Symmetric groupoids, Mat. Sb. 31 (1952), 136-151 (in Russian).

16. G. Mashevitzky And B. M. Schein, Automorphisms of the endomorphism semigroup of a free monoid or a free semigroup, Proc. Am. Math. Soc. 131 (2003), 1655-1660.

17. G. Mashevitzky, B. Plotkin and E. Plotkin, Automorphisms of categories of free algebras of varieties, Electron. Res. Announc. Am. Math. Soc. 8 (2002), 1-10.

18. M. Petrich, Inverse semigroups, Pure and Applied Mathematics (Wiley Interscience, 1984).

19. M. Petrich And N. R. Reilly, Completely regular semigroups (Wiley, 1999).

20. M. Petrich And P. V. Silva, Relatively free bands, Commun. Alg. 28 (2000), 26152631.

21. L. PolÁk, A solution of the word problem for free *-regular semigroups, J. Pure Appl. Alg. 157 (2001), 107-114.

22. I. SchrEIER, Über Abbildungen einer abstrakten Menge auf ihre Teilmengen, Fund. Math. 28 (1936), 261-264.

23. H. SEzinando, The cardinalities of the Green classes of the free objects in varieties of bands, Semigroup Forum 44 (1992), 164-198.

24. R. P. Sullivan, Automorphisms of transformation semigroups, J. Austral. Math. Soc. 20 (1975), 77-84. 